\title{
Uma arquitetura para Hipermídia Adaptativa baseada em recursos da Web Semântica
}

\author{
Sandro José Rigo ${ }^{1,2}$, Éverton Eckert Schneider ${ }^{1}$, José Palazzo M. de Oliveira ${ }^{2}$ \\ Universidade do Vale do Rio dos Sinos (UNISINOS) São Leopoldo - RS - Brasil \\ rigo@unisinos.br, everton.schneideregmail.com \\ Instituto de Informática - Universidade Federal do Rio Grande do Sul (UFRGS) \\ Porto Alegre - RS - Brazil \\ palazzo@inf.ufrgs.br
}

\begin{abstract}
The cognitive overload is one of the undesired effects of the web development. Hypermedia systems can help to solve this problem, by generating personalized results. This paper describes an architecture for Adaptive Hypermedia applications based on Semantic Web resources. The main goal of this proposal is to observe and describe the advantages that could be obtained with a semantic based application description and with improved interaction possibilities. The high flexibility to generate adaptations and facilities for semantic content annotation are some of the advantages of this architecture.
\end{abstract}

Resumo. A sobrecarga cognitiva é um dos efeitos não desejados que resultam do grande desenvolvimento da Web. Sistemas de Hipermídia Adaptativa possibilitam minimizar este problema, proporcionando ao usuário resultados personalizados. Este documento descreve uma arquitetura para aplicações de Hipermídia Adaptativa baseada em recursos da Web Semântica. O principal objetivo da proposta é observar vantagens que podem ser obtidas a partir da descrição da aplicação com recursos de semântica e com possibilidades maiores de interação. Algumas destas são a flexibilidade para a geração de adaptações e facilidades para a anotação semântica de conteúdos.

\section{Introdução}

A Internet está em crescimento há vários anos, fato que vêm gerando uma demanda pela personalização de conteúdos das páginas Web, visando evitar a sobrecarga cognitiva [1] existente atualmente. Esta melhoria pode ser obtida com técnicas de Hipermídia Adaptativa, a partir das quais são relacionadas informações sobre o domínio da aplicação com informações sobre o perfil de usuários, o que permite a geração de resultados mais adequados ao contexto de uso para cada usuário ou grupo de usuários.

A Hipermídia Adaptativa aborda o desenvolvimento de sistemas capazes de promover a adaptação de conteúdos e recursos hipermídia oriundos de diversas fontes (banco de dados, documentos na Internet, serviços Web) e apresentados em diversos formatos (por exemplo, texto, áudio, imagens). Esta adaptação se dá em relação às 
informações conhecidas de um determinado usuário, que descrevem suas preferências, objetivos, conhecimentos prévios e necessidades. É possível encontrar aplicação direta para esta abordagem em áreas como educação, sistemas de informação para ajuda online, comércio eletrônico e marketing, entre outros. O resultado almejado é proporcionar a cada usuário uma interface onde estilo, conteúdo, recursos e hyperlinks são apresentados conforme seus objetivos e preferências [1,2]. Este resultado pode ser obtido com técnicas como a apresentação adaptativa ou a navegação adaptativa, com o objetivo de adaptação de conteúdo e de adaptação de hyperlinks, respectivamente.

Apesar de serem conhecidos estudos sobre área de Hipermídia Adaptativa desde a década de 90 [3], alguns trabalhos mais recentes sinalizam uma falta de padrões para a área, o que pode levar a problemas de interoperabilidade. Estes podem ser solucionados com o uso de recursos da Web Semântica, cujo papel é permitir que sistemas computacionais possam realizar melhor uso dos dados presentes na Web [4]. Um destes recursos são as ontologias, usadas como uma forma de representar conhecimento sobre domínios diversos e descrevendo normalmente indivíduos (instâncias), classes (conjuntos, coleções, tipos de objetos), atributos (propriedades, características), relações e eventos (mudança de atributos ou relações) $[5,6]$. Ontologias podem ser representadas com linguagens tais como OWL ${ }^{1}$. Além de ontologias a Web Semântica permite a descrição de informações sobre recursos com outras linguagens, tais como, por exemplo, com a linguagem $\mathrm{RDF}^{2}$, que provê uma especificação padrão para descrição de metadados sobre recursos na Web.

Em geral as aplicações de Hipermídia Adaptativa são descritas por modelos que indicam, por exemplo, informações do domínio, apresentação de conteúdos ou características de usuários. As adaptações são geradas com base em regras e na aquisição de informações do usuário. A utilização dos recursos da Web Semântica neste contexto possibilita a descrição de modelos mais flexíveis e abrangentes, de modo a facilitar a tarefa de adaptação [7, 8]. Entretanto uma dificuldade observada na aplicação de recursos de Web Semântica é a inerente necessidade de especialização dos usuários responsáveis pela geração das relações, o que torna proibitivo a utilização em larga escala, por exemplo, de editores de ontologias ou a anotação semântica.

Este trabalho descreve uma arquitetura para aplicações de Hipermídia Adaptativa que incorpora recursos da Web Semântica. A utilização destes permite maior flexibilidade na descrição dos modelos para domínio da aplicação e apresentação de conteúdos. A proposta também facilita a interação dos usuários responsáveis pela geração de conteúdo Web, de modo que estes não precisem de conhecimentos profundos sobre tecnologias ou linguagens de desenvolvimento para Internet e nem sobre publicação de conteúdo na Web ou sobre Web Semântica. Deste modo um dos principais diferenciais do trabalho consiste na abordagem privilegiando o usuário responsável pela criação e manutenção do conteúdo, evitando a necessidade de conhecimento aprofundado da área de Web Semântica. O outro diferencial do trabalho consiste em uma abordagem simplificada para a descrição e utilização de modelos

\footnotetext{
${ }^{1}$ http://www.w3c.org/owl

${ }^{2}$ http://www.w3c.org/rdf
} 
necessários para a geração de adaptações. Foi utilizado um conjunto de ontologias que permite a descrição da estrutura do site Web e os formatos gerais de apresentação de conteúdos, que podem ser relacionados a partir de regras baseadas no comportamento e nas informações do perfil dos usuários. Por fim, o trabalho foi desenvolvido de forma que as adaptações não necessitem da identificação pessoal dos usuários, em uma abordagem onde a identificação de classes de comportamento a partir das sessões típicas dos usuários origina a informação necessária para as adaptações. Todo o processamento destas informações é realizado continuamente e integrado ao funcionamento do sistema, sem necessidade de etapas adicionais para geração de padrões.

Este trabalho está organizado como segue. Na seção dois são descritos e analisados alguns trabalhos relacionados ao tema aqui desenvolvido. A seção três realiza o detalhamento da arquitetura desenvolvida, indicando detalhes dos recursos da Web Semântica que foram utilizados. A seguir, a seção quatro descreve uma experiência de utilização da arquitetura em um caso prático de publicação e acesso a um site Web. Por fim, a seção cinco expõe as conclusões alcançadas.

\section{Análise de trabalhos relacionados}

Vários sistemas adaptativos foram desenvolvidos sem a utilização de recursos da Web Semântica, sendo baseados em modelos e regras. Nestes casos observam-se algumas dificuldades em relação à geração das adaptações, que poderiam tornar-se mais flexívieis, por exemplo, com o uso de inferências ou com o compartilhamento de informações (por exemplo, do perfil de usuários). O AHA(Adaptive Hypermedia Architecture) [9] mantém um modelo de usuários baseado em conhecimento sobre conceitos e oferece recursos de adaptatividade de conteúdo e hyperlinks através do uso de fragmentos inseridos no código $\mathrm{HTML}^{3}$. O sistema Elena PLA [10], com foco principal no suporte para personalização em ambiente distribuído, utiliza modelo de usuários, repositórios de conteúdos, serviços de anotação e de recomendação.

A aplicação da linguagem $\mathrm{XML}^{4}$ para a descrição de estruturas e conteúdos, indicações de anotações ou descrição de perfil de usuários pode ser acompanhada a partir de exemplos como AdaptWeb [11], ambiente adaptativo para aprendizagem, onde são observados o programa de aprendizado, o conhecimento do aluno e suas preferências de interação. Nele pode-se observar a interação de recursos como a anotação semântica, a descrição de estrutura de conteúdos e a adaptação de características dos conteúdos, de acordo com o contexto do usuário. Um ambiente de autoria permite a descrição de metadados associados ao material sendo publicado e uma descrição do modelo do usuário é utilizada a partir de uma ontologia, prevendo operações de personalização.

Outros sistemas baseados em recursos da Web Semântica foram desenvolvidos a partir de abordagens inicialmente descritas sem propósito de adaptação, tal como a proposta do método ASHDM (Adpative Semantic Hypermedia Design Method) [15], descrito a partir do SHDM (Semantic Hypermedia Design Method) [12], método de

\footnotetext{
${ }^{3}$ http://www.w3c.org/MarkUp

${ }^{4} \mathrm{http}: / /$ www.w3c.org/XML
} 
modelagem que busca representar tanto um projeto conceitual como projeto navegacional utilizando linguagens de descrição de ontologias, possibilitando o desenvolvimento de sites Web a partir de características semânticas.

Alguns exemplos tratam da integração de recursos de Web Semântica e Hipermídia Adaptativa. O OntoWeaver [13] é uma proposta baseada em ontologias para desenvolvimento de sites na Web, incluindo funcionalidades de adaptação. Utiliza-se uma ontologia de visualização, para modelar a estrutura de páginas Web e a interface do usuário, e uma ontologia de apresentação, definida para descrever os estilos de apresentação e os formatos para as páginas [14]. Realiza a adaptação de informações com um modelo de usuários e um modelo de customização baseados nas preferências de cada usuário. O OntoWebber [16] utiliza ontologias para composição da interface a partir dos seguintes elementos: "cards", "links" e "pages". Um elemento "page" integra os demais elementos, que podem ser dinâmicos, utilizando mecanismo de inferência TRIPLE [17]. A modelagem de personalização permite a identificação de recursos adequados para grupos ou para usuários, com características e propriedades indicando interesses e condições de navegação desejadas. A proposta do SEAL [18] também é baseada em ontologias e voltada para a descrição de portais Web. As etapas para a construção destes portais Web são o projeto de ontologia, integração de dados, projeto de site Web e implementação. Para o projeto de site envolve a construção do modelo de navegação, modelo de entrada e modelo de personalização. Para sua implementação é utilizado o framework $\mathrm{KAON}^{5}$, que possui ferramentas integradas para a descrição de ontologias, integração de dados e geração personalizada do portal, a partir da identificação de usuários e seu contexto.

Desta forma, observa-se que a utilização de ontologias para a descrição de estruturas, modelos e informações do perfil de usuários facilita a geração de adaptações e sua manutenção. Entretanto estas construções tendem à geração de uma estrutura de difícil manuseio pelos usuários responsáveis pela edição dos conteúdos. Em geral as adaptações são geradas com base no conhecimento de preferências de usuários, demandando a sua identificação.

\section{Detalhamento da arquitetura desenvolvida}

A arquitetura proposta tem como objetivo apresentar aos usuários páginas com características adaptativas em um ambiente que utiliza recursos da Web Semântica. Foi tomada como premissa a geração de adaptações sem a identificação do usuário. Para isso as informações de uso são aproveitadas como base, junto com informações de perfis de grupos de usuários obtidos com o acompanhamento do uso. São usadas duas ontologias, uma contendo informações e hierarquia das páginas pertencentes ao site Web (Ontologia da Aplicação) e a outra com a definição da interface gráfica que cada página pode possuir (Ontologia de Apresentação). O sistema mantém uma base de dados para os conteúdos das páginas, que armazena todos os conteúdos em formato textual, relacionando-os a partir da Ontologia da Aplicação e permitindo representar diferentes conteúdos para uma mesma página, com informações que podem ser usadas

\footnotetext{
${ }^{5} \mathrm{http} / / /$ kaon.semanticweb.org/
} 
na tarefa de adaptação. Todos os acessos dos usuários são mantidos de forma resumida em uma base de dados. As regras de adaptação baseiam-se na utilização do site e na estrutura descrita nas ontologias. Elas relacionam informações do perfil do usuário com a estrutura do site, indicando possibilidades de adaptação utilizadas em conjunto com informações descritas na Ontologia de Apresentação. As possibilidades de adaptação implementadas foram definidas como acréscimos à estrutura do site, na forma de hyperlinks adicionais, e como ajustes de conteúdos, na forma de exibição de material adicional.

Para proporcionar flexibilidade na implementação da arquitetura, a mesma foi separada em dois módulos: validação e adaptação. Seu funcionamento geral, descrito na Figura 1, a seguir, pode ser resumido em etapas como: recebimento de solicitação de acesso; consulta da página solicitada na Ontologia da Aplicação; gravação da informação correspondente ao modelo do usuário; consulta, na Ontologia de Apresentação, do template correspondente à página acessada; montagem do resultado intermediário (ainda sem adaptação) em código HTML; verificação do padrão comportamental através do modelo do usuário; caso tenha sido encontrado o perfil do usuário é feita a aplicação de regras de adaptação, caso contrário é utilizado o formato padrão; geração do código final (com adaptações) em codificação HTML; envio da resposta para o usuário.

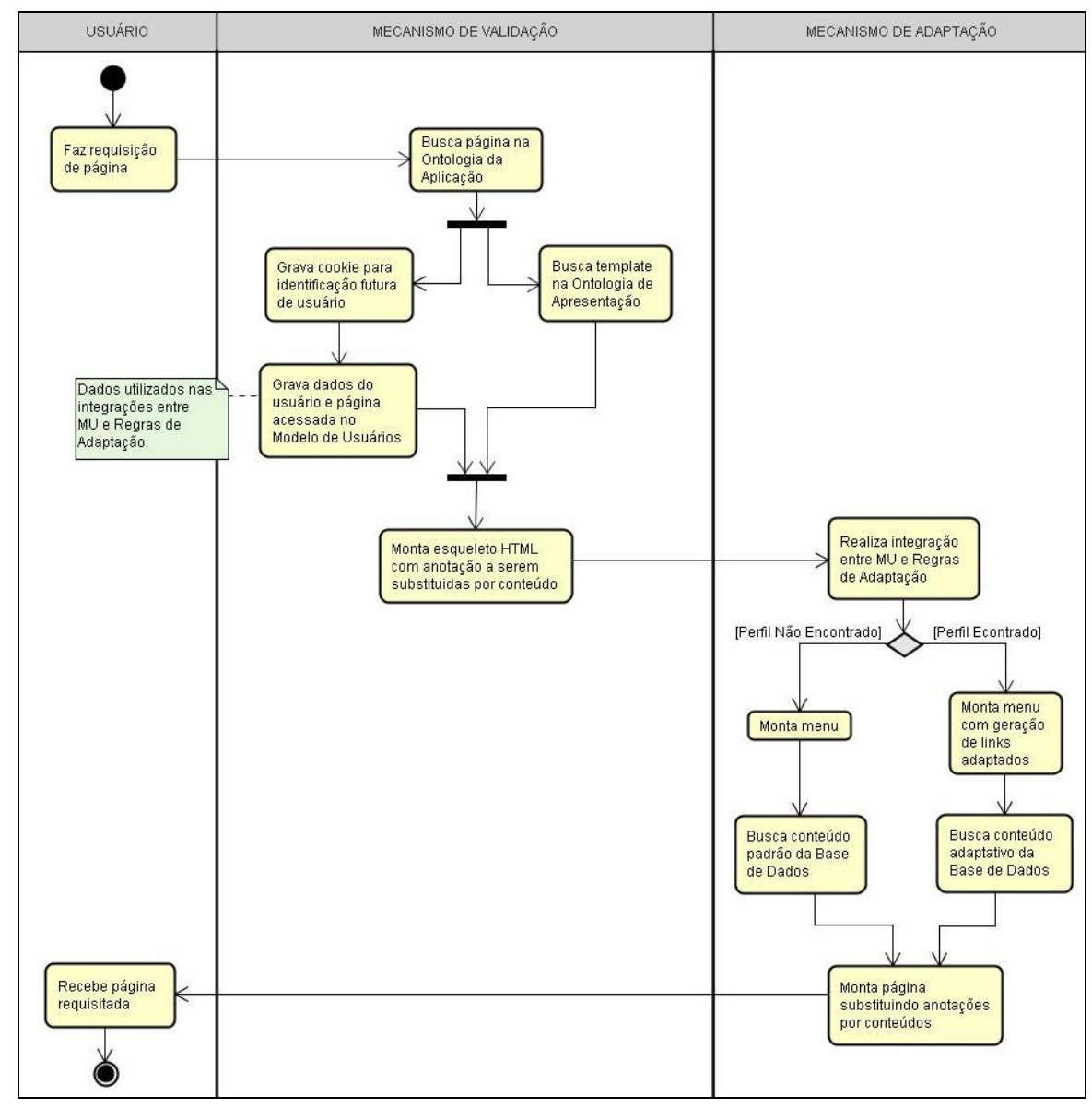

Figura 1: Funcionamento geral da arquitetura desenvolvida 
O mecanismo de validação é responsável pela montagem inicial do código HTML da página requisitada e pela gravação de informações que possibilitam adaptações de conteúdo em requisições futuras. Utiliza-se um cookie para identificar acessos da sessão de um usuário. Esta abordagem foi escolhida por facilitar a identificação de informações da sessão, apesar de sua desvantagem no caso de desativação junto ao navegador do usuário. Cada acesso gera um registro que compõe o modelo de usuários, contendo dados sobre a navegação, tais como a página acessada e o tipo de conteúdo. Algumas destas informações são geradas pela requisição recebida e outras são complementadas com uma consulta à ontologia da aplicação. Nela estão descritas a estrutura da aplicação e os detalhes de cada página, permitindo a categorização do acesso com informações semânticas como o tipo de conteúdo e as relações desta página com as demais.

Estas ontologias foram descritas manualmente por especialistas no domínio da aplicação, com uso do editor de ontologias Protege ${ }^{6}$, tendo sido utilizada a linguagem $\mathrm{OWL}^{7}$ para sua representação. Não foram empregadas ontologias já existentes, para proporcionar ao trabalho maior liberdade de experimentação.

\subsection{Ontologia da Aplicação}

A Ontologia da Aplicação é uma ontologia criada dinamicamente, ou seja, a cada página criada, atualizada ou removida no site, essa ontologia sofre alterações, mantendo-se sempre com a estrutura de páginas do portal Web atualizada. Parte da Ontologia da Aplicação está descrita brevemente na Figura 2, na qual podem ser observadas algumas relações, como a "possuiSubpagina". Além destas relações são mantidos alguns atributos definidos nesta ontologia, como "tipoConteudo" e "template", e outros reutilizados de outras terminologias conhecidas, como o Dublin Core ${ }^{8}$, no caso de "dc:description" e "dc:creator". A figura ilustra algumas instâncias, identificadas pela relação "io", de um possível site Web.

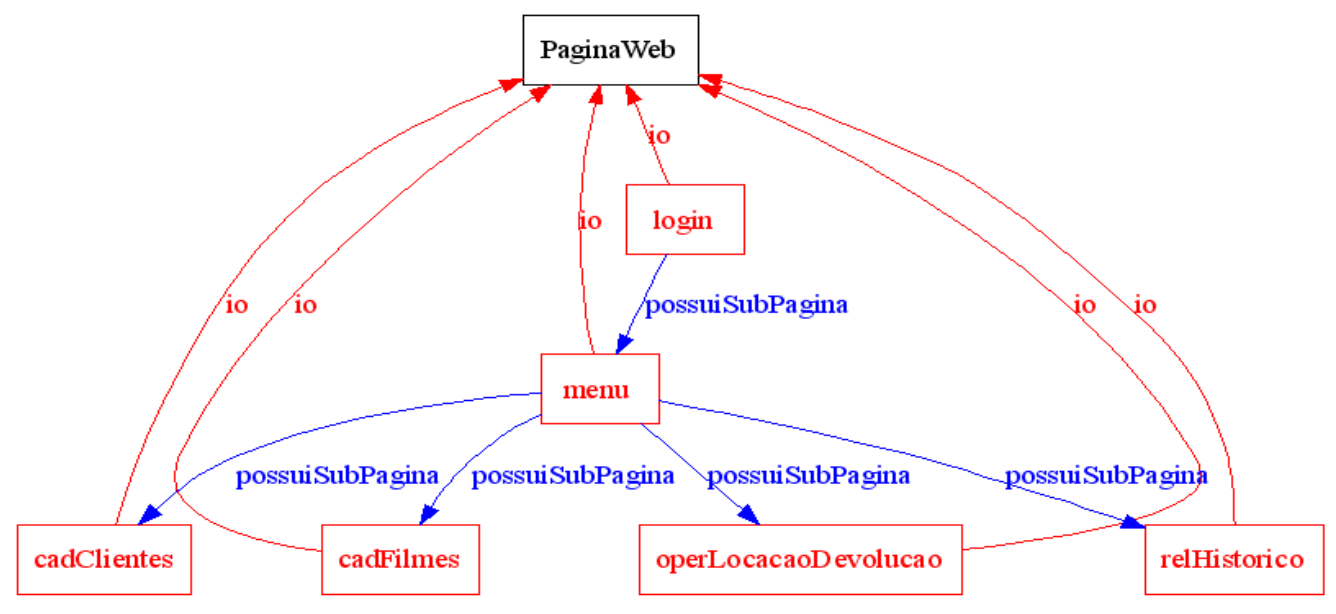

$6 \mathrm{http}: / /$ protege.stanford.edu

7 http:// www.w3.org/2004/OWL

$8 \mathrm{http}: / /$ dublincore.org/ 
Figura 2: Trecho da estrutura da Ontologia da Aplicação

A Figura 3 ilustra parcialmente o código da ontologia em OWL, onde são apresentados os elementos citados anteriormente.

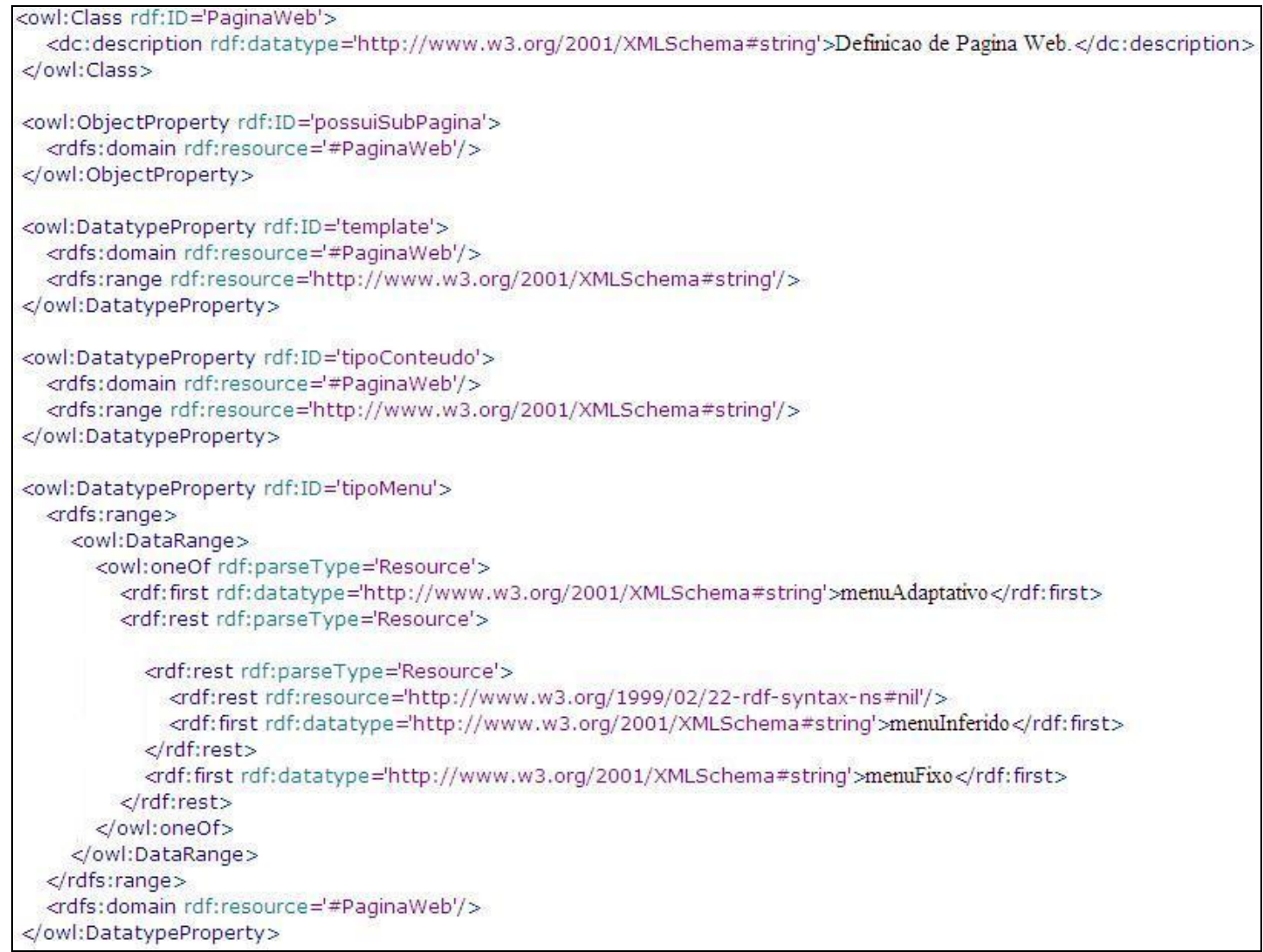

Figura 3: Código parcial dos elementos da Ontologia da Aplicação

A cada página cadastrada ou alterada no site a Ontologia da Aplicação é atualizada com os dados alterados ou com um novo registro referente à página. A Figura 4 apresenta o código da ontologia gerado para descrever a página "Menu".

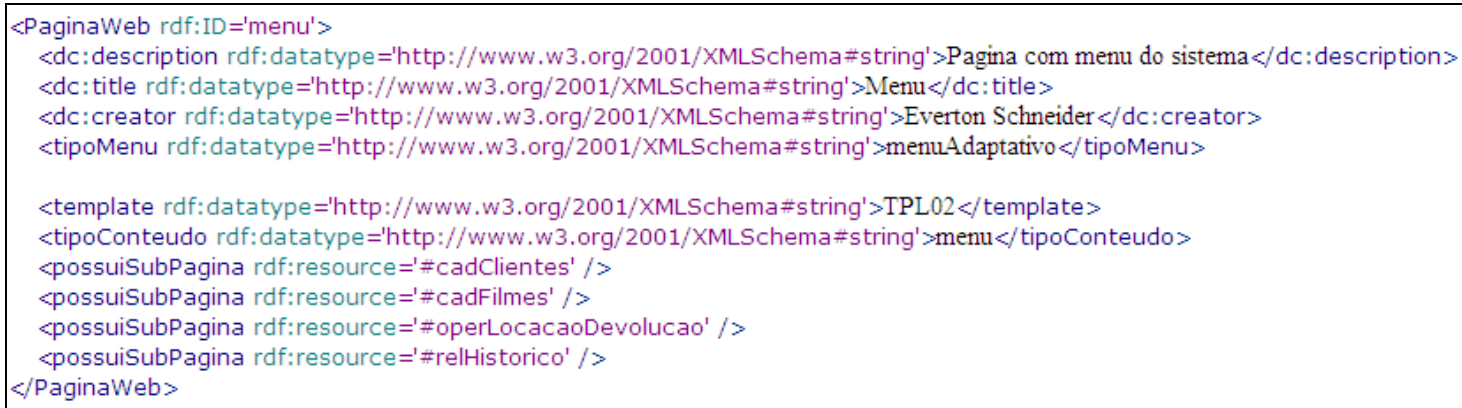

Figura 4: Código para representar uma página na Ontologia da Aplicação

\subsection{Ontologia de Apresentação}

A Ontologia de Apresentação é uma ontologia estática, não alterada pelo usuário ou pela arquitetura. Possui a função de descrever características da interface a ser definida. Cada elemento desta ontologia indica especificamente um tipo de formato, no 
elemento "template". Estes formatos são descritos, na Ontologia de Apresentação, a partir dos conceitos "template", "elemento" e "sub-elemento", que permitem a descrição de dados da página e possibilitam a geração do código HTML básico com anotações de conteúdo para substituições. Nas seções onde devem ser feitas substituições por conteúdos adaptados são utilizados marcadores específicos. A Figura 5 ilustra a descrição de uma típica área de interface, descrita por uma instância do tipo "template", identificada como "TPL01conteudo". Ela é composta por uma instância do tipo "elemento", identificado como "TPL01 conteudo_div", que descreve informações para a geração do código HTML (através do elemento "tag"). Por sua vez, esta instância é composta por um "sub-elemento", o que é indicado com a relação "possuiSubElemento" para com a instância identificada como "TPL01 conteudo_pre", que possui informações para geração de substituição de conteúdo (através do marcador "swah:text"). Estas informações são utilizadas em conjunto com outros elementos da codificação padrão necessária para a montagem de uma página em HTML.

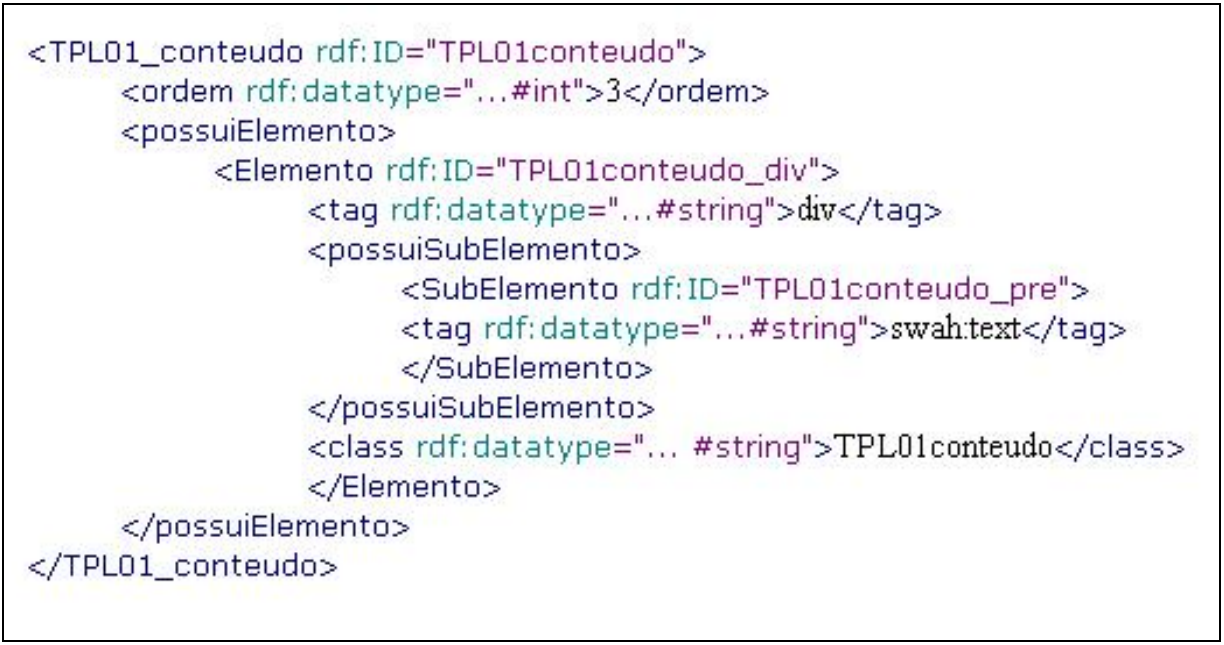

Figura 5: Parte da ontologia de apresentação

\subsection{Banco de dados}

A Ontologia da Aplicação armazena basicamente a lista de páginas criadas em um portal Web, enquanto a Ontologia de Apresentação descreve o leiaute que cada página possuirá ao ser apresentada ao usuário. Os dados das páginas são armazenadas em um banco de dados MySQL, onde os dados de cada área definida na Ontologia de Apresentação precisam ser cadastrados. Esse cadastro pode ser realizado através da interface de administração SWAH, apresentada na seção 3.7.

\subsection{Modelo de usuários}

O modelo de usuários tem a necessidade de armazenar características de acesso dos usuários, que posteriormente podem ser usadas para adaptação de conteúdos. $\mathrm{O}$ armazenamento dos dados referentes ao modelo de usuários da arquitetura é feito em um conjunto de tabelas do banco de dados, o que facilita a recuperação dessas informações. 
A cada acesso o usuário é identificado através de um cookie gravado na sua máquina. Para cada acesso a arquitetura verifica se o usuário possui o cookie de identificação, se esse já existe a arquitetura utiliza essa identificação para gravar os registros no modelo de usuários. Caso o usuário que está acessando a página ainda não possua esse cookie, a arquitetura armazena um novo cookie na máquina cliente e esse usuário é identificado como um usuário que ainda não acessou as páginas anteriormente.

No momento que o usuário acessa uma página controlada pela arquitetura, automaticamente seu acesso está sendo gravado. A página que está sendo acessada, a data e hora do acesso, assim como tipo de conteúdo vinculado a página são as informações presentes no modelo de usuários.

\subsection{Regras de adaptação}

Cada página utilizada pela arquitetura tem um conteúdo padrão para as suas áreas (topo, conteúdo e outros), que são inseridos caso nenhuma regra de adaptação cruzada com o modelo de usuários seja satisfeita. Além desses conteúdos padrão existem também os conteúdos adaptativos, que recebem um nome que os identifica para que sejam usados em adaptações de conteúdo nas páginas de acordo com regras satisfeitas na relação entre regras e modelo de usuários.

Para inferências entre regras e modelo de usuários não foi localizada nenhuma biblioteca em linguagem PHP que automatizasse o processo, assim foi criada uma estrutura em XML para a armazenagem das mesmas. A utilização do XML possibilita flexibilidade para a manipulação das regras e também facilita a inclusão de regras automaticamente, etapa prevista em trabalhos futuros. Inicialmente foi verificada a possibilidade do uso de $\mathrm{SWRL}^{9}$, porém com a falta de recursos para a inferência com mecanismos que suportam a linguagem PHP, essa linguagem foi usada somente para o embasamento da estrutura XML de regras da arquitetura. Dessa forma, foram criadas regras baseadas em condições e ações, separadas em três tipos.

- Regras de menu: regras onde são cadastrados links que serão sugestionados no menu.

- Regras de perfil: regras para definir um perfil através de uma lista de acessos por tipos de conteúdo da página.

- Regras de conteúdo: regras que identificam para cada perfil um conteúdo destinado a uma das áreas da página (topo, conteúdo e outros).

\subsubsection{Regras de menu}

As regras de menu são extremamente simples e definem um conjunto de links que serão sugestionados para um usuário. A arquitetura não leva em consideração o perfil do usuário para aplicação dessas sugestões. Essas regras são criadas com uma condição (tipo de conteúdo da página que está sendo acessada) e " $n$ " ações (páginas que serão sugestionadas). A Figura 6 apresenta um trecho de código XML para demonstrar a

\footnotetext{
${ }^{9}$ SWRL: Semantic Web Rule Language (linguagem de regras para a Web Semântica)
} 
utilização desse tipo de regras, onde para o acesso da página com o tipo de conteúdo "livrosTecnicos" a arquitetura apresentará links para as páginas com nome "tabacariaRevistasTecnicas", "tabacariaRevistasInformativas" e "tabacariaDvdsFilmes".

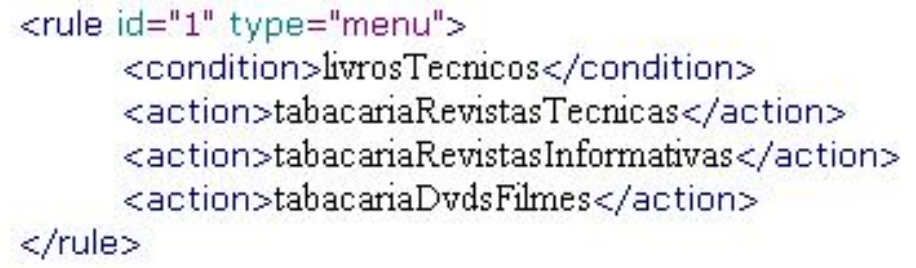

Figura 6: Regra de menu

\subsubsection{Regras de perfil}

As regras de perfil têm o objetivo de definir um perfil com base nos tipos de conteúdo acessados pelo usuário. Mais de uma página pode conter o mesmo tipo de conteúdo cadastrado, como por exemplo uma página com uma lista de geladeiras e outra página com uma lista de microondas, que podem ter o tipo de conteúdo "eletrodomésticos". Esse tipo de regras não está associado com ações específicas, sendo formado somente por condições. Sua utilização leva em conta a navegação do usuário e a similaridade dos tipos de páginas da sessão de navegação com os tipos de páginas indicados na regra. Sempre que houver uma similaridade acima de um limiar arbitrário, será realizada uma adaptação. O trecho de código da Figura 7 identifica um perfil, chamado "infantil", que está condicionado ao acesso das páginas com tipo de conteúdo "LivrosInfantis", "RevistasInfantis" e "DvdsJogos". Sempre que uma página é acessada a arquitetura faz uma contagem de quantas vezes as páginas citadas em cada perfil, definido nas regras, foram acessadas pelo usuário, criando uma classificação interna dos perfis e associando um valor a cada um deles. O perfil com maior valor é considerado o perfil do usuário. As regras de perfil podem ser criadas com liberdade e sem limitação de número máximo de perfis, assim pode até mesmo existir uma especialização de características dos perfis, com regras mais amplas e outras mais restritas.

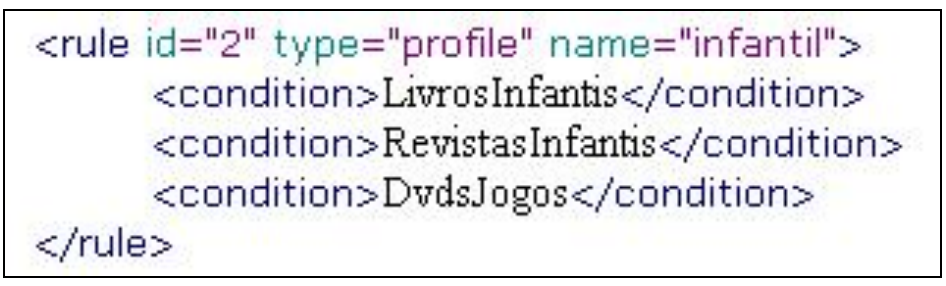

Figura 7: Regra de perfil

\subsubsection{Regras de conteúdo}

Regras de conteúdo são as regras que serão utilizadas pela arquitetura para a adaptação dos conteúdos. Elas relacionam um tipo de perfil aos conteúdos adaptativos cadastrados na base de dados para inserir esses conteúdos nas áreas dos templates das páginas. Este é o tipo mais complexo de regra, pois existem sempre duas condições (perfil e página acessada) e uma ação para cada área da página definido no seu template, que é descrito na Ontologia de Apresentação. A Figura 8 ilustra a regra de conteúdo para uma página com nome "tabacariaLivrosInfantis", onde para o perfil do tipo "tecnico" os 
dados serão apresentados da seguinte forma: a área do topo receberá a imagem com o tipo de conteúdo "topLivrosInfantis_PerfilTecnico" (atributo type=“top"); a área de conteúdo receberá o conteúdo cadastrado com o nome "contentLivrosInfantis_PerfilTecnico" (atributo type="content"); a área de conteúdos alternativos receberá o conteúdo com o nome "otherLivrosInfantis_PerfilTecnico" (atributo type="other").

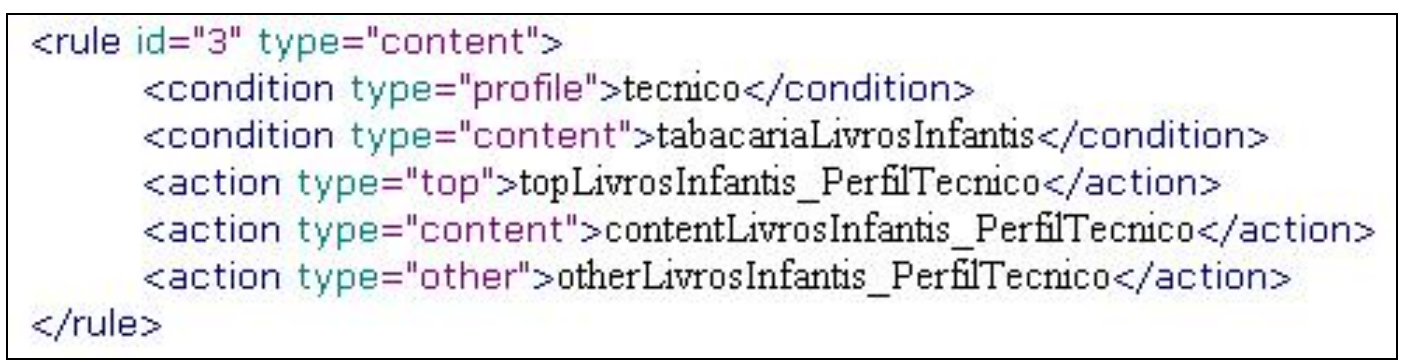

Figura 8: Regra de conteúdo

O processo de montagem das regras não é difícil, porém bastante trabalhoso, tendo em vista que cada uma das páginas com características adaptativas precisará de regras cadastradas para cada um dos perfis definidos que desejem adaptações, ou pelo menos para os perfis para os quais serão necessárias adaptações. Este processo será melhorado, em trabalhos futuros, com a geração e inclusão automática de regras.

\subsection{Fluxo de informações e as adaptações}

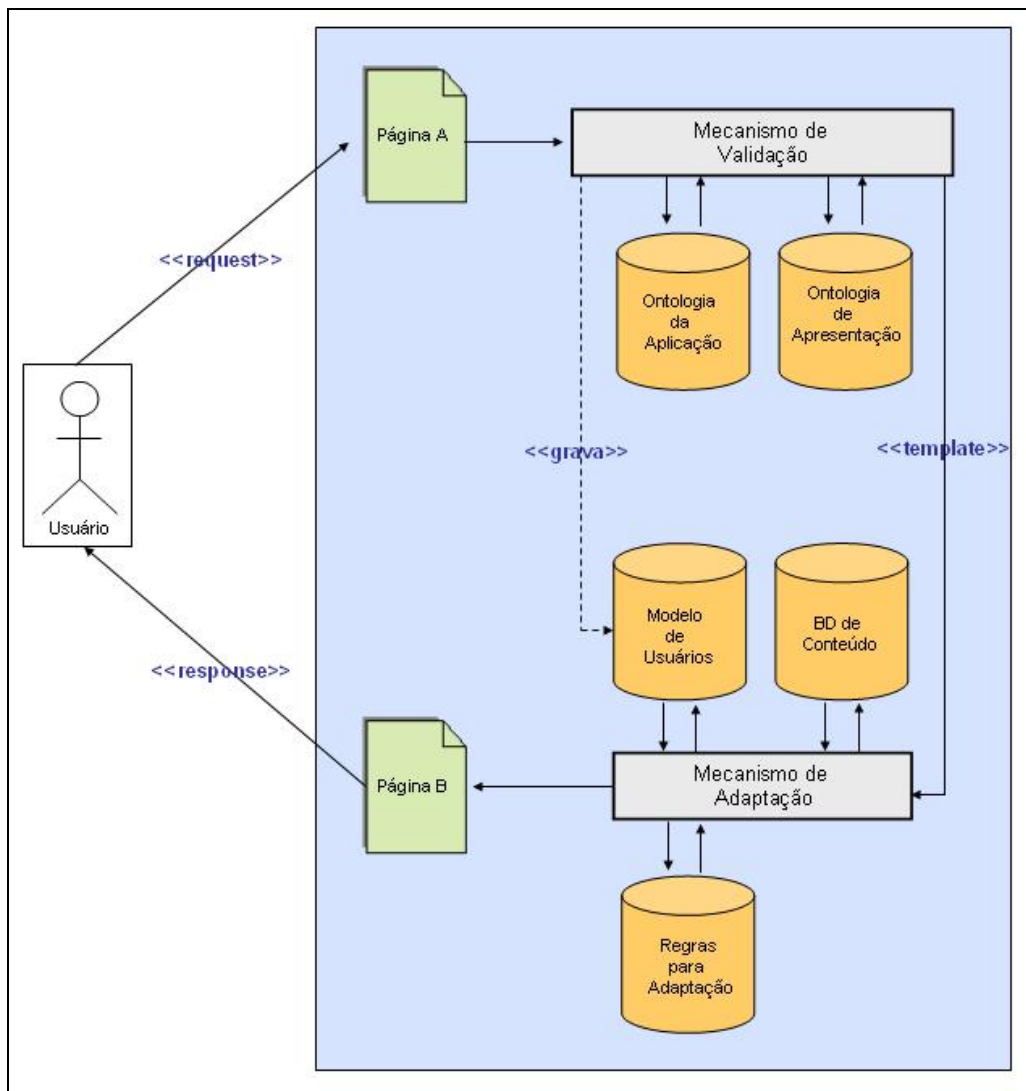

Figura 9: Fluxo de dados na arquitetura 
O fluxo de dados é iniciado quando o usuário faz a requisição de acesso a uma página. No momento que a página é requisitada, a arquitetura verifica sua existência na Ontologia da Aplicação, que liga a página a um template, descrito na Ontologia de Apresentação. Ao realizar o acesso da página é gravado imediatamente um registro no modelo de usuários que será usado na identificação do seu perfil. O resultado do mecanismo de validação é um código HTML que representa o esqueleto da pagina, sem suas informações, somente com anotações que serão substituídas. O mecanismo de adaptação realiza a análise da codificação HTML gerada pelo mecanismo de validação e substitui suas anotações por conteúdos. Para determinar os conteúdos correspondentes a cada anotação é feita uma análise entre o modelo de usuário e as regras de adaptação, em busca de um padrão adequado. Caso nenhuma regra válida seja encontrada, a página a ser apresentada ao usuário será montada com seu conteúdo padrão.

\subsection{Interface de administração - SWAH}

Para facilitar a criação e publicação de páginas por usuários sem conhecimentos em tecnologias da Web Semântica, foi desenvolvida uma ferramenta de administração de conteúdos criados através da arquitetura, denominada SWAH (Semantic Web / Adaptive Hypermedia). A interface disponibiliza telas de cadastros de páginas e inserção de conteúdos, telas para upload de imagens e regras, além de telas de configuração geral dos parâmetros do ambiente. A Figura 10 exibe algumas telas do ambiente de administração. No item 1 desta figura está exemplificado o menu de opções disponível ao usuário e no item 3 é possível verificar o resultado de uma pesquisa das páginas cadastradas, a partir do qual é possível a edição das páginas desejadas.

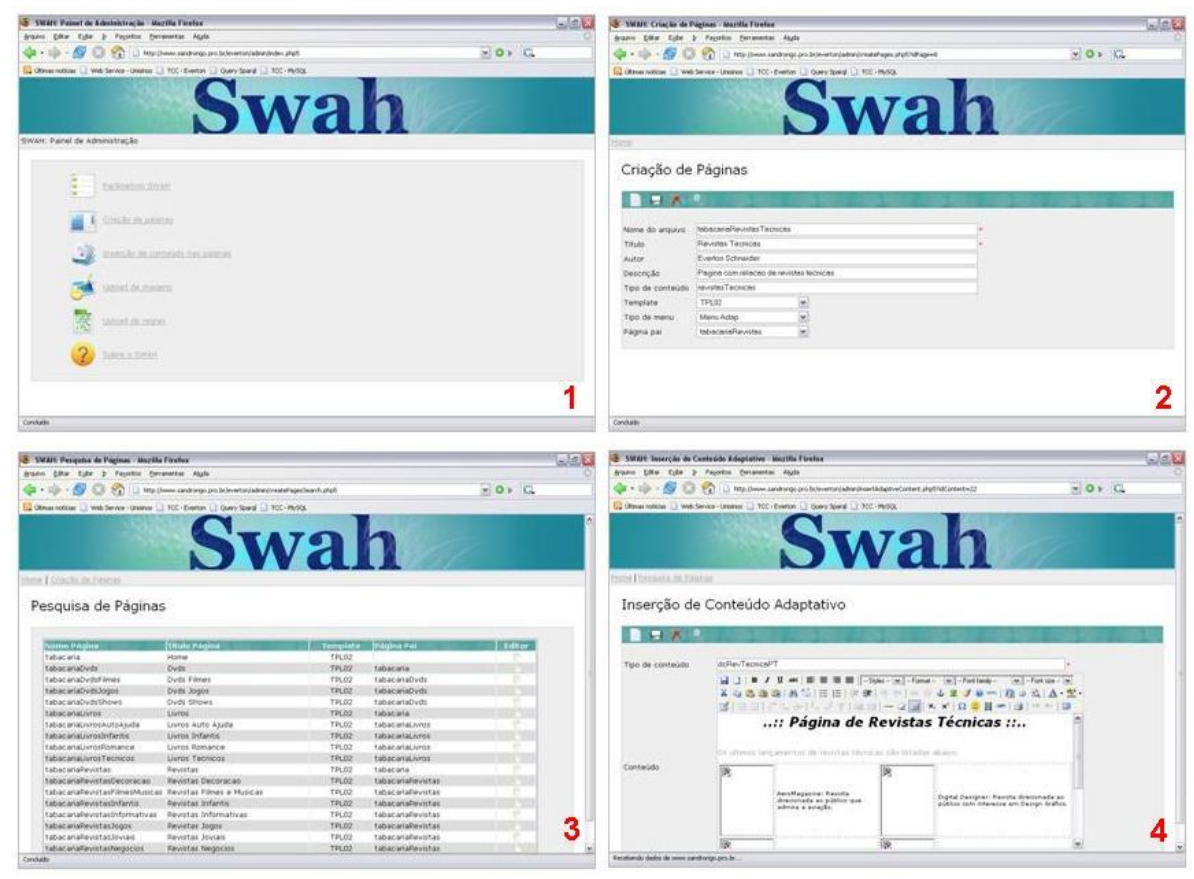

Figura 10: Telas da interface SWAH

O item número 2 da Figura 10 apresenta a tela de criação de páginas, que mantém a Ontologia da Aplicação atualizada com cada página atualizada pelo usuário. Nesta tela constam informações de cadastro como nome do arquivo, título da página, 
autor, descrição e tipo de conteúdo, template escolhido (descrito na Ontologia de Apresentação), tipo de menu a ser utilizado (adaptativo, inferido ou fixo) e página superior na hierarquia (página "pai”). Após o cadastro de uma página é necessário inserir conteúdo para a mesma. $\mathrm{O}$ item número 4 da Figura 10 mostra a tela de inserção de conteúdos, onde é editado o conteúdo padrão da página, ou seja, conteúdo que é apresentado caso não existam regras de adaptação para a mesma. Nesta tela podem ser observadas as informações gerais obtidas da tela anterior, no cadastro da página, seguida pela área de edição do conteúdo onde está disponível uma barra de ferramentas que busca facilitar a interação entre os usuários e a edição de páginas. No caso da inserção de conteúdo adaptativo, além destas informações é indicado um nome para o tipo de conteúdo da página (que também deverá ser usado na montagem das regras), bem como inserido o conteúdo correspondente. Os dados cadastrados pela interface SWAH são armazenados em um banco de dados $\mathrm{MySQL}^{10}$, sendo que o acesso às ontologias é realizado com uso da biblioteca $\mathrm{RAP}^{11}$, sendo utilizada a linguagem PHP $5^{12}$ para a implementação dos sistema.

\section{Exemplo de utilização}

Para a verificação prática da aplicabilidade das funcionalidades implementadas na arquitetura foi desenvolvido um caso de uso com base nos requisitos para uma tabacaria, possuidora de três categorias de produtos: livros, revistas e DVDs. Devido à facilidade de relacionamento de áreas entre essas categorias, foram feitos testes com regras para sugestão de hyperlinks no menu de páginas distintas e com a substituição ou complementação de conteúdos.

\begin{tabular}{|l|l|}
\hline \multirow{4}{*}{ Item } & \multicolumn{1}{|c|}{ Categoria } \\
\hline \multirow{4}{*}{ DVDs } & Filmes \\
\cline { 2 - 2 } & Jogos \\
\cline { 2 - 2 } & Shows \\
\hline \multirow{4}{*}{ Livros } & Auto-Ajuda \\
\cline { 2 - 2 } & Infantil \\
\cline { 2 - 2 } & Romance \\
\cline { 2 - 2 } & Técnico \\
\hline \multirow{4}{*}{ Revistas } & Decoração \\
\cline { 2 - 2 } & Filmes \\
\cline { 2 - 2 } & Infantil \\
\cline { 2 - 2 } & Informativas \\
\cline { 2 - 2 } & Jogos \\
\cline { 2 - 2 } & Negócios \\
\cline { 2 - 2 } & Técnicas \\
\hline
\end{tabular}

Tabela 1: Páginas cadastradas para a experimentação

\footnotetext{
${ }^{10} \mathrm{http}: / /$ www.mysql.com/

${ }^{11} \mathrm{http} / / /$ sites.wiwiss.fu-berlin.de/suhl/bizer/rdfapi/

$12 \mathrm{http}: / /$ www.php.net/
} 
Para o caso de uso foram criados quatro perfis de usuários, identificados como adulto, jovial, infantil e técnico. A primeira etapa para a criação do site foi o cadastro de suas páginas, tendo sido criadas catorze páginas, todas relacionadas às três categorias de produtos citadas. Foram criadas várias subcategorias para distinguir os tipos de produtos, como por exemplo, livros técnicos, revistas infantis, DVDs de filmes, entre outros. A seguir foram inseridos os conteúdos das páginas e criados conteúdos adaptativos. A tabela 1, acima, apresenta as páginas criadas e suas respectivas categorias.

Cada página criada através da arquitetura para o site de experimentação teve um conteúdo padrão cadastrado, além de um conteúdo relacionado a cada um dos quatro perfis criados (infantil, jovial, adulto e técnico). A Figura 11 apresenta a página de livros técnicos com a apresentação de seu conteúdo padrão, enquanto na Figura 12 é possível visualizar a mesma página com seu conteúdo adaptado para um usuário que a arquitetura identificou como usuário de perfil "técnico".

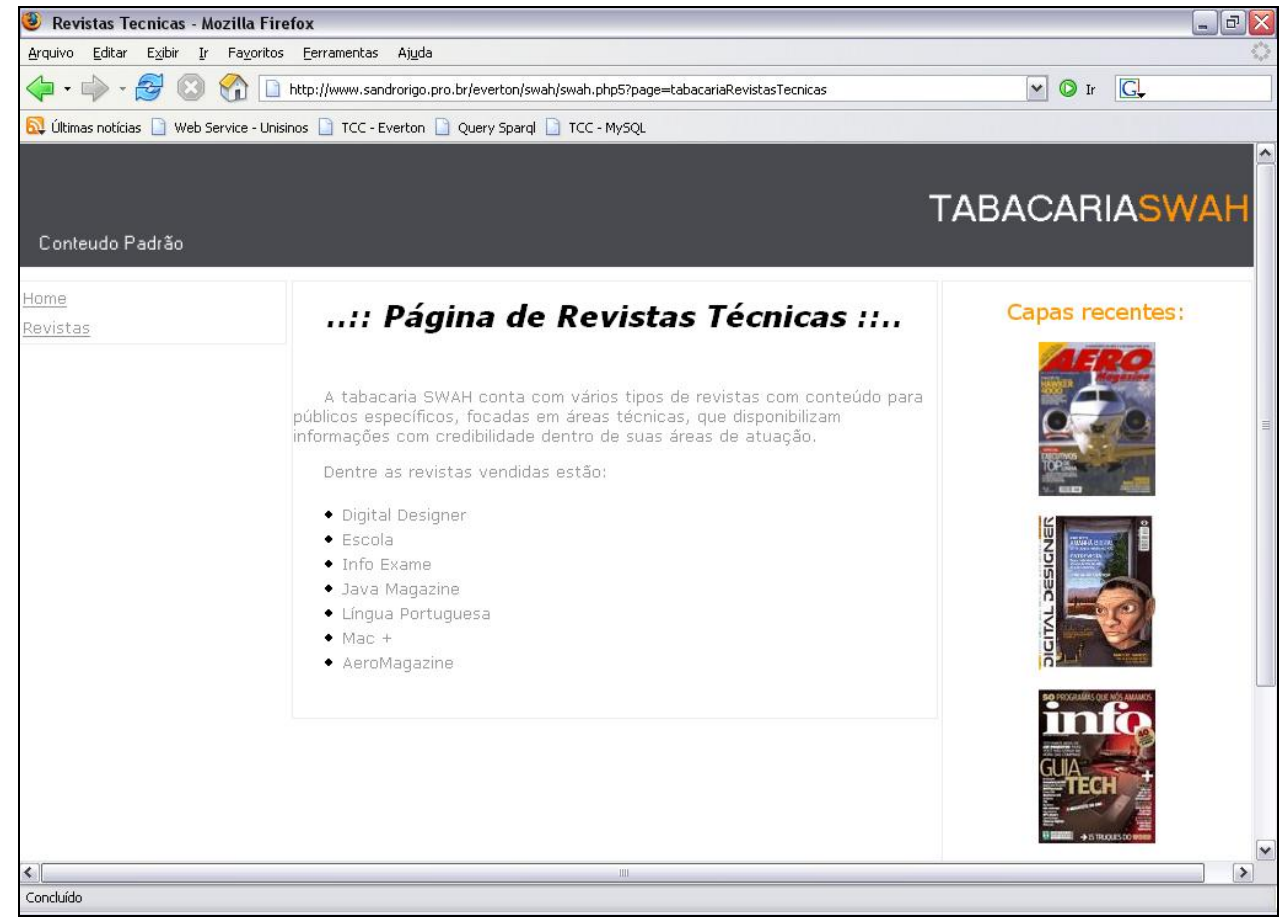

Figura 11: Página com conteúdo padrão

Os exemplos apresentados nas Figuras 11 e 12 são páginas de revistas técnicas. A página da Figura 11 não possui adaptações, estando com seu conteúdo padrão em todas suas áreas. A página apresentada na Figura 12 está com todas suas áreas adaptadas devido às regras de adaptação e o comportamento do usuário em sua navegação entre as páginas do portal, o que resultou na avaliação de seu perfil como perfil técnico. Segundo as regras criadas para a o site de experimentação, um usuário de perfil técnico acessa com maior freqüência categorias como livros de auto-ajuda e livros técnicos, revistas de filmes, jogos e de assuntos técnicos, além de DVDs de jogos. Estas informações podem 
ser observadas na Figura 13, onde a regra descrita com ao atributo type='profile' e name='tecnico' descreve estas possibilidades de adaptação.

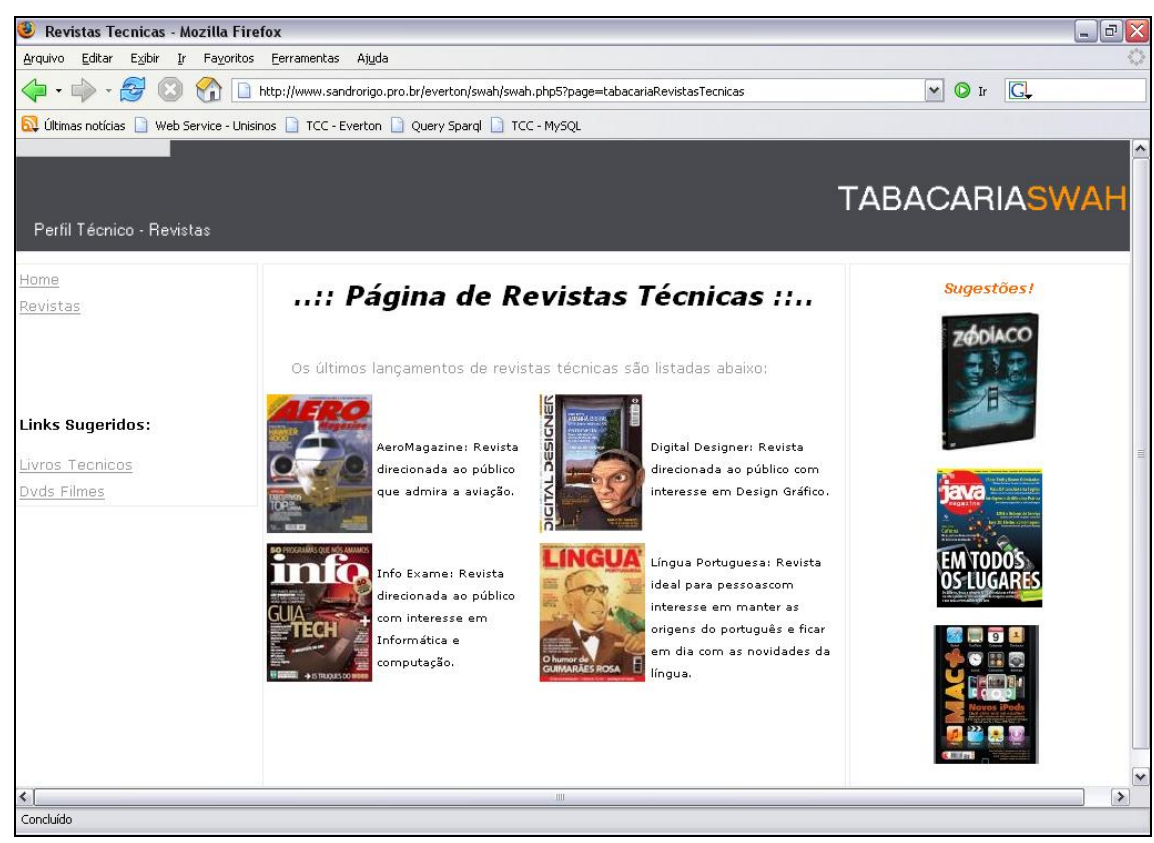

Figura 12: Página com conteúdos adaptados

As regras cadastradas podem ser verificadas na Figura 13. É importante observar a indicação dos conteúdos adaptativos nas regras com atributo type='content'. A regra indica que se o usuário possui o perfil técnico e estiver acessando a página com nome "tabacariaRevistasTecnicas", o topo deve usar o conteúdo "topRevTecnicasPT", a área central o conteúdo "dcRevTecnicaPT" e a área lateral o conteúdo "ocRevTecnicaPT". Esses nomes são atribuídos aos conteúdos na sua criação, através da interface de administração, na tela de inserção de conteúdos adaptativos. 


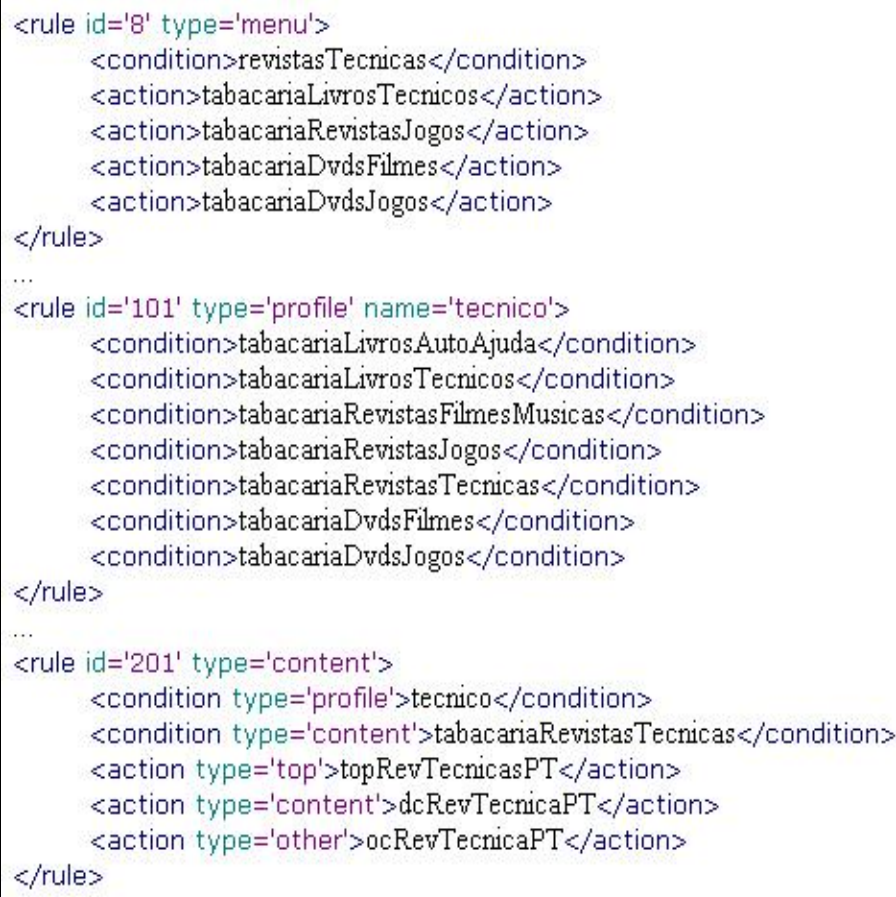

Figura 13: Regras para adaptação de conteúdo

Também é possível ver na Figura 13 a adaptação de hyperlinks do menu de acordo com as regras anotadas com atributo type='menu'. As regras de menu indicam que, ao acessar a página com nome "revistasTecnicas", sejam mostrados os hyperlinks das páginas de livros técnicos, revistas de jogos, DVDs de filmes e DVDs de jogos. A arquitetura SWAH possibilita esse tipo de comportamento para qualquer página criada com base em sua estrutura, utilizando suas ontologias e regras de adaptação.

\section{Conclusões}

O sistema apresentado permite a descrição de um site web a partir de dois modelos armazenados em ontologias e implementa o acompanhamento de sua utilização. Este acompanhamento possibilita a utilização de regras de adaptação, baseadas na informação semântica descrita nas ontologias. Assim é possível a geração de adaptações de estrutura e de conteúdo, sem necessidade de identificação de usuários individualmente, a partir de tarefas realizadas em uma sessão do usuário. Além disso, o sistema permite ao usuário responsável pela geração e manutenção de conteúdo um acesso facilitado e não requer o conhecimento de conceitos de Web Semântica, o que facilita a sua adoção.

Foi realizada a utilização do sistema para a montagem de um site Web e a realização de testes de uso. A partir desta experiência observaram-se indícios de facilidades para a edição de conteúdos, pois o responsável pela geração dos conteúdos não possui necessidade de conhecimentos específicos em Web Semântica, tais como necessários para a descrição de ontologias. Também foi observado que o mecanismo de adaptações, a partir dos diferentes formatos de regras, permite a customização do resultado em diversos aspectos, possibilitando a geração de resultados personalizados. 
Dentro do contexto do trabalho várias oportunidades de melhorias e novas implementações apareceram como possibilidades de se tornar trabalhos futuros. Dentre as melhorias seria interessante proporcionar maiores facilidades à edição de conteúdos e às regras de adaptação, assim como uma interface que possibilite a manutenção e criação de novos templates que fiquem armazenados na Ontologia de Apresentação, de modo a completar o ciclo de ferramentas para que o sistema possa ser completamente utilizado por usuário sem conhecimento em Web Semântica ou desenvolvimento Web. O desenvolvimento de um mecanismo que possa identificar o comportamento do usuário e gerar automaticamente regras por agrupamento de perfis poderia ajudar a arquitetura na adaptação de conteúdos, evitando a manutenção manual das regras de adaptação pelos administradores do portal Web criado. A adaptação de conteúdo com base em perfis estáticos, onde existiria a necessidade de identificação do usuário, pode ser considerada uma alternativa para o amadurecimento da arquitetura, pois possibilitaria a inclusão de questionários em páginas para identificar a evolução dos usuários na utilização do sistema. Por fim, seria interessante a geração de mapas adaptativos, onde seja possível visualizar o mapa do site com conteúdos adaptativos relacionados a cada página, resultando em um mapa com todas as possibilidades de adaptação das páginas existentes no portal Web.

\section{Referências}

[1] Brusilovsky, P., Karagiannidis, C., and Sampson, D. (2004) Layered evaluation of adaptive learning systems. International Journal of Continuing Engineering Education and Lifelong Learning 14 (4/5), 402 - 421.2004.

[2] De Bra, Arroyo, L., Chepegin, V., The next big thing: adaptive web-based systems. Journal of Digital Information, V(5)N(1). 2004.

[3] Brusilovsky, P. "Adaptive hypermedia". User Modeling and User-Adapted Interaction, 11:87-110, 2001.

[4] Kravčík, M., Gašević, D.. Adaptive hypermedia for the semantic web. Conference on Hypertext and Hypermedia. Pages 3-10, Odense, Dinamarca, 2006.

[5] Freitas, F. L. G., "Ontologias e a Web Semântica”. XXIII Congresso da Sociedade Brasileira de Computação. JAI. Campinas, São Paulo, Junho de 2003.

[6] Fensel, D. Ontology-Based Knowledge Management. IEEE Computer, 35(11) pages 56-59, 2002.

[7] De Bra, Arroyo, L., Chepegin, V., The next big thing: adaptive web-based systems. Journal of Digital Information, $\mathrm{V}(5) \mathrm{N}(1) .2004$.

[8] Aroyo, L., et al. Ontology-based personalization in user-adaptive systems. 2nd International Workshop on Web Personalization, Recommender Systems and Intelligent User Interfaces (WPRSIUI'06), 2006.

[9] BRA, Paul De, CALVE, Licia. AHA: A Generic Adaptive Hypermedia System. 2nd Workshop on Adaptive Hypertext and Hypermedia, Pittsburg, EUA, 20/06/1998 - 24/06/1998.

[10] Dolog, P. et al. Personalization in distributed e-learning environments. In Proceedings of WWW2004 - The Thirteen International World Wide Web Conference, New York, May 2004. P.85-94. ACM Press.

[11] Oliveira, J. P. M., Muñoz, L., "Adaptative web-based courseware development using metadata standards and ontologies". CAise conference, Riga, Latvia, June 7-11, 2004.

[12] LIMA. Fernanda. Modelagem semântica de aplicações na WWW. Rio de Janeiro: PUC, Departamento de Informática, 2003.

[13] Ontoweaver - An ontology based approach to web site design and development. Disponível em: $<$ http://kmi.open.ac.uk/projects/akt/ontoweaver>. Acessado em: 14 jun 2007.

[14] LEI, Y., MOTTA, E., DOMINGUE, J.. Modelling Data-Intensive Web Sites with OntoWeaver. In Proceedings The International Workshop on Web Information System Modelling (WISM 2004), pages pp. 106-121, Riga, Latvia. 
[15] Assis, P. S., Schwabe, D. Nunes, D. A.,: ASHDM - Model-Driven Adaptation and Meta-adaptation. Adaptive Hypermedia and Adaptive Web-Based Systems, 4th International Conference, AH 2006, Dublin, Ireland, June 21-23, 2006, Proceedings. Lecture Notes in Computer Science 4018 Springer 2006.

[16] Jin, Y., Xu, S., and Decker, S. Ontowebber: Model-driven ontology-based web site management. (SWWS 2001), pages 529-547. Stanford University.

[17] Sintek, M. and Decker, S. (2002). Triple - an rdf query, inference, and transformation language. In First International Semantic Web Conference (ISWC 2002), volume 2342 of Lecture Notes in Computer Science, p. 364-378. Springer.

[18] Maedche, A., Staab, S., Stojanovic, N., Studer, R., and Sure, Y. (2003). Semantic portal: The seal approach. In Spinning the Semantic .pages 317-359. MIT Press. 